\title{
CROSS-CULTURAL ASPECT OF INTERPERSONAL RELATIONSHIPS IN THE WORKPLACE IN THE PERIOD OF REORGANIZATION
}

\author{
Konstantinov Vsevolod ${ }^{1}$, Shumilkina Evgeniia ${ }^{2}$, \& Osin $\operatorname{Roman}^{3}$ \\ ${ }^{I}$ General Psychology department, Penza State University, (Russia) \\ ${ }^{2}$ Head of research department, Penza State University (Russia) \\ ${ }^{3}$ Associate Professor of the Department of General Psychology, Penza State University (Russia)
}

\begin{abstract}
In the conditions of fragility of building interethnic relations, turning to the problem of development of interpersonal relations in mono-cultural and multi-cultural teams of employees of an enterprise in the period of reorganization is extremely relevant. In order to improve the efficiency of organizations great attention is paid to the role of cross-cultural management in forming the effective intercultural interaction and reducing intercultural conflicts. The article presents the results of the empirical research conducted by the authors; the conclusions made after processing data using the methods of mathematical statistics. The analysis of the obtained empirical data shows that in the period of reorganization the factor of cross-cultural composition of the teams of employees under study actively manifests itself in interpersonal relations. Differences were found in the level of certain characteristics of employees in different types of ethnic environments. In general, more statistically significant connections between personal and behavioral characteristics were found in the sample of employees in a multi-ethnic environment compared to the employees in a mono-ethnic environment. The development of interpersonal interaction in a team of employees in a multi-ethnic environment in the period of reorganization should be based on the development of the most significant characteristics of their personality and behavior: positive ethnic identity, empathy, interpersonal trust and skills and abilities of building interpersonal interaction.
\end{abstract}

Keywords: Russia, interpersonal relationships, cross-cultural communication, multi-ethnic environment, quality of relationships at work, labor collectives, interethnic relations.

\section{Introduction}

At present the issues of cross-cultural management and intercultural communications are becoming more and more relevant: business and intercultural relations are expanding, multinational teams are being formed. Among the theories that describe the essence of cultural differences and explain their impact on organizational behavior, the following concepts are distinguished: Values Orientation Theory (Kluckhohn \& Strodtbeck, 1961); Context of Culture (Hall \& Hall, 1990); Cultural Dimensions Theory (Hofstede, Hofstede, \& Minkov, 2010; Konstantinov, 2017). Differences between cultures in the attitude to the world were empirically confirmed. There are individualistic (competitive relations and each worker's values are at the heart of management) and collectivist (hierarchically built models of management, values of the team are more important) cultures (Bono \& Yoon, 2012; Ryzhova, Konstantinov, Gritsenko, \& Khukhlaev, 2018; Khukhlaev et al., 2019; Konstantinov \& Kovaleva, 2013).

At first, in the 1970s the world smaller countries (Finland, Denmark, Sweden, and the Netherlands) started to study the problems of cross-cultural differences in international business. Later these countries were joined by the leading world powers (Germany, Great Britain, the USA), and later on by Italy, Spain, and France.

R. Lewis emphasizes the fact that in the context of globalization modern business requires both great knowledge and good relationships, it is necessary to take into account cross-cultural aspects (Lewis, 2000). At the same time, problems in intercultural communication arise not because of the difficulties of communication, but because of the differences of individuals.

The quality of interpersonal relationships determines the way employees behave both at work and in personal life (Allen \& Eby, 2012; Dutton, 2014). Typically, high-quality relationships lead, among other things, to their commitment, productivity, motivation, innovation, error detection, favorable employee behavior, teamwork, helping others, effective internal and external organizational communication, avoiding conflicts, and resistance to negative events. Conversely, poor employee relationships have a detrimental impact on these aspects of the organization (Bono \& Yoon, 2012; Ryzhova et al., 2018; Khukhlaev et al., 2019; Konstantinov \& Kovaleva, 2013). The level of efficiency 
and development of an organization is largely determined by the ability of employees to interact and cooperate with each other, which can be traced in a cross-cultural context.

The impact of the ethno-cultural factor, which leads to the formation of a special environment for building interpersonal relations, was studied by a number of domestic and foreign psychologists. But the data on the influence of ethnic characteristics of individuals on their interpersonal relationships are insufficient to build a holistic view of this process (Berry, Galyapina, Lebedeva, Lepshokova, \& Ryabichenko, 2019; Grigoryev, van de Vijver, \& Batkhina, 2018; Khukhlaev, Kuznetsov, \& Chibisova, 2013; Raman, Sambasivan, \& Kumar, 2016).

Interpersonal relationships are subjectively experienced relationships between people, mutually influencing each other in the process of cooperation and communication. They differ in a number of parameters: their origin, stability, duration, openness of the parties, etc. The process of initiation, maintaining and termination of interpersonal relationships is determined by a number of factors. These include individual psychological and typological features of the interpersonal interaction, as well as the conditions for this interpersonal interaction.

The ethnic factor is an important factor for maintaining efficient interpersonal relations. Researchers note that the ethnic environment includes many modifications of the surrounding reality, accumulated by members of the ethnic community in the course of its historical development. The ethnic factor sets the context for interpersonal relations, determines the behavioral patterns of their individuals and the readiness to maintain relations with a member of another ethnic group. At the personal level, ethnicity is manifested in the level of the person's ethnic identity, ethnic self-consciousness, the ability to acquire ethnic attitudes, stereotypes, prejudices, etc.

\section{Selection, techniques and methods of the research}

426 employees (from 18 to 56 years of age) of enterprises of the Penza region and the Republic of Mordovia participated in our empirical study. We assumed that interpersonal relationships of employees in the period of reorganization are a complex phenomenon determined by the ethnotype of the working environment and characterized by a number of features that influence their qualitative and quantitative parameters.

Verification of the proposed hypotheses was carried out by solving a number of theoretical, methodological and empirical problems. The empirical study aimed to compare the distribution of status positions among members in mono- and multi-ethnic groups, to identify the level of conflict in their relations, to study the examples of the phenomena of empathy and trust in mono - and multi-ethnic environment, to establish the type of the relationship between ethnic identity and response/feedback strategies in conflict situations (compromise, cooperation).

In the course of the study, the following methods and techniques were used: "The Interpersonal Trust Scale" by J. B. Rotter, "The technique of empathic abilities diagnosis" by V. V. Boyko, "Types of ethnic identity" by G. U. Soldatova, "The level of conflict" by A. M. Ganeev and L. S. Tronova, "The features of handling conflict styles" by C. Thomas. To process the results of the study, mathematical procedures of indicators and statistical data reliability assessment were used (Raygorodsky, 2017; Tatarko \& Lebedeva, 2011).

\section{Results of the research, their discussion}

In the course of the empirical study, it was found that employees of mono-ethnic professional environments are much more likely $(\mathrm{p}<0.01)$ to come into contact with each other than workers of multi-ethnic professional environments. Contacts of people belonging to different ethnic groups are usually limited to issues connected with business or job responsibilities.

Workers in mono-ethnic environments maintain interpersonal contacts for a longer period than workers in multi-ethnic environments $(\mathrm{p}<0.05)$. Interpersonal relations of workers in mono-ethnic groups are more dynamic by nature and prolonged in time. Considerable length of interpersonal contacts in mono-ethnic environments is caused by numerous reasons and grounds to establish and maintain such relationships, by greater interest in each other, their desire to communicate, and their openness to interaction.

There is a difference in the modality of interpersonal relationships between the workers of the two samples (at a statistically reliable level of $\mathrm{p}<0.01$ ). Workers from a mono-ethnic professional environment show more enthusiasm than workers in multi-ethnic environments. These observations indicate that in multi-ethnic environments there is certain tension and reticence in contacts and interpersonal relations between employees of different ethnic groups. At the same time, they are more sensitive to issues concerning ethnicity and ethnic background, so they are more likely to engage in conflict with each other.

The percentage of workers with the average status in mono- and multi-ethnic environments is approximately the same $(75.9 \%$ and $70.4 \%$, respectively). Differences were found in the percentage of 
workers with high and low sociometric status depending on the environment they belong to. The percentage of high status workers in mono- and multi-ethnic professional environments was $19.1 \%$ and $11.7 \%$ respectively $(\mathrm{p}<0.05)$. More fundamental differences were found in the subsamples of workers with low sociometric status in mono- and multi-ethnic environments $(5.0 \%$ and $17.9 \%$ respectively). The percentage difference is characterized by statistical significance $(p<0.01)$, i.e. in multi-ethnic environments the proportion/ percentage of workers with low sociometric status is much higher than in mono-ethnic environments.

In mono-ethnic groups the number of workers with a low level of conflict exceeds $(18.1 \%)$ the proportion of such workers in multi-ethnic environments $(13.1 \%)$. The same trend is observed with the percentage of workers who are characterized by an average level of conflict: there are slightly more of them in mono-ethnic groups $(68.2 \%)$ than in multi-ethnic groups $(61.9 \%)$. The level of statistical significance $\mathrm{p}<0.05$, indicating certain differences between the compared samples, does not allow us to note their stability.

According to the results, workers in multi-ethnic professional environments show a higher level of conflict than workers in mono-ethnic environments. The reason is that the multi-ethnic environment requires that workers meet additional challenges, e.g. choosing life priorities. In contrast to workers from mono-ethnic environments, who are faced only with the task of personal self-assertion, professional and life choices, workers from multi-ethnic environments need to self-identify in their own ethnic environment, to correlate their personal, professional, and life priorities with ethnic attitudes, norms, and customs. At the same time, they have to interact with representatives of other ethnic groups, with an ethnically different culture, attitudes and customs. The complexity and diversity of the tasks, workers from multi-ethnic environments are faced with, create tension; make them more aggressive and contentious in interpersonal interaction. To the greatest extent, it is this kind of behavior that is used with people belonging to other ethnic groups.

Workers from mono-and multi-ethnic environments have statistically significant differences ( $p<0.01)$ in the degree of manifestation of the high and low levels of interpersonal trust. In the sub-samples of workers, there was a significant excess of the number of employees with a high level of interpersonal trust (the average value is $27.0 \%$ ) over the number of employees with a low level of interpersonal trust $(14.0 \%)$. In sub-samples of workers in multi-ethnic environments, a statistically significant excess of the number of employees with a low level of interpersonal trust $(27.6 \%)$ over the number of employees with a high level of interpersonal trust (13.3\%) was found. In a sample of workers working in a mono-ethnic environment, the average value of interpersonal trust is 6.5 units, in a sample of workers in a multi-ethnic environment it is 4.3 units. The obtained discrepancy in the quantity of manifestation of the trait indicates statistically significant differences in its manifestation in workers, depending on what environment (mono- or multi-ethnic) the employee belongs to. In a multi-ethnic environment, interpersonal trust between workers is much less manifested than among workers working in a mono-ethnic environment.

In the sample of workers from a multi-ethnic environment, there tends to be more workers with a low level of empathy. However, the differences in the level of empathy were somewhat more smoothed, not so vividly manifested. This can be explained by the specific functioning of the phenomena under consideration. It is obvious that empathy has more opportunities for its development, regardless of the ethnic and national characteristics of the people around. Trust is based on knowledge of a wider range of background information that characterizes the subject of potential interaction. Accordingly, it may be easier for a person to show empathy than interpersonal trust. At the same time, it is interpersonal trust that has the greatest influence on the quality and intensity of building interpersonal relationships in the environment.

In multi-ethnic environments in all sub-samples tested by the "Types of ethnic identity" by G. U. Soldatova, the proportion of workers characterized by ethno-egoism, which can be expressed either in a harmless form or by a very aggressive behavior, predominates. In the first case, the perception of the surrounding world through the semantic prism of "my people" comes first. In the second case, employees demonstrate sufficient tension/aggression in interpersonal relationships with people belonging to other ethnic groups. It can be supposed the high level of conflict and low levels of empathy and interpersonal trust in multi-ethnic environments is caused the dominance of this type of ethnic identity.

Studying the types of response to a conflict situation allowed us to find out that in the samples of workers in mono-ethnic environments, cooperation is the most widely-used strategy of behavior in conflict situations $(27.5 \%)$. This type of behavior manifests itself in adopting the decision that fully satisfies the interests of both parties to the conflict. Trying to achieve a compromise is another type of widespread behavior in a conflict of employees in a mono-ethnic environment $(23.6 \%)$. The behavior of employees who follow this pattern of behavior is manifested in the establishment of an agreement between the parties to the conflict. Competition/rivalry is another form of behavior in a conflict situation $(22.2 \%)$. This type of behavior in a conflict situation is manifested in the desire of workers to forward their own interests at the expense of the interests of the partners in interpersonal interaction. In the samples of workers under study adaptation (14.4\%) and avoidance behavior $(12.3 \%)$ come next. These 
strategies of behavior in a conflict situation are chosen by approximately $10 \%$ of the entire sample of employees in a mono-ethnic environment.

In a sample of workers in multi-ethnic environments other behavioral preferences in conflict situations were detected. However, we note that the preferred behavioral patterns in the sample of employees were distributed more evenly in this group. The evident type of behavior in the sample of workers in a multi-ethnic environment was avoidance behavior (23.8\%). Accordingly, we can say that approximately $25 \%$ of the employees, in this case, are focused on avoiding a conflict situation. At the same time, they do not demonstrate the desire to ensure cooperation with the subject of interaction and are not focused on achieving any of their own goals in a conflict situation. Approximately the same percentage of employees in the sample of employees in a multi-ethnic environment was focused on the strategy of competition in a conflict situation (23.1\%). This type of behavior, associated with the desire to achieve the satisfaction of their interests at the expense of the interests of the partner in interpersonal relations, was demonstrated by about a quarter of all employees in a multi-ethnic environment. In a multi-ethnic environment the strategy of adaptation to the current situation was mostly expressed. This type of behavior was chosen by $21.6 \%$ of workers, i.e. about $1 / 5$ of the entire sample. This type of behavior is characterized by sacrificing one's own interests for the sake of the interaction partner. This behavior is the opposite of rivalry/competition in a conflict situation, which is the second most commonly demonstrated behavioral pattern in this sample. The cooperation strategy (20.6\%) turned out to be significant for employees in a multi-ethnic environment. This type of behavior in a conflict situation was a priority for about $1 / 5$ of the workers in the sample. This suggests that a fairly large proportion of workers in a multi-ethnic environment are ready to accept an alternative that would fully satisfy the interests of both sides in interpersonal relations.

The most evident differences between workers in mono- and multi-ethnic environments were revealed in the two types of behavior in a conflict situation - trying to achieve a compromise and avoidance behavior. Workers who study in a mono-ethnic environment are more inclined to use the first type, while workers in a multi-ethnic environment tend to follow the second behavioral pattern. A difference in the propensity to use adaption strategy and to cooperate was revealed only as a trend. The first type of behavior (adaption) is characteristic of workers in a multi-ethnic environment, while the second (cooperation) characterizes workers in a mono-ethnic environment.

The analysis of the obtained empirical data shows that the factor of cross-cultural composition of the groups has a great impact on interpersonal relationships in their teams under study in the period of reorganization. The method of correlation analysis made it possible to establish differences in the level of intensity of interconnection of employees belonging to different types of ethnic environments. In mono-ethnic environments several trends characterizing their interpersonal relationships are observed: trust goes with a tendency to compromise $(\mathrm{p}<0.001)$; ethnic nihilism is directly combined with conflict, on the one hand, and with a tendency to compromise, on the other hand, $(\mathrm{p}<0.01)$; ethnic nihilism goes with ethnic fanaticism (feedback) $(\mathrm{p}<0.01)$; ethnic indifference is combined with a tendency to cooperate (feedback) $(\mathrm{p}<0.05)$; avoidance behavior is not compatible with the tendency of an individual to compete in a conflict situation of interpersonal interaction $(\mathrm{p}<0.01)$.

In the multi-ethnic environment the following relationships were observed: those between a positive ethnic identity and a tendency to cooperate (direct link) $(\mathrm{p}<0.01)$, as well as tendency to ethnic isolationism and conflict (feedback) $(\mathrm{p}<0.001)$; empathy and a person's desire to cooperate (direct link) and ethnic isolationism (feedback) $(\mathrm{p}<0.01)$; interpersonal trust and inclination to adapt (direct link) $(\mathrm{p}<0.01)$; a tendency to compromise and avoidance behavior (feedback) $(\mathrm{p}<0.05)$. The development of interpersonal interaction in a multi-ethnic environment in a team of employees in the period of reorganization should be based on the development of the most significant characteristics of their personality and behavior: positive ethnic identity, empathy, interpersonal trust and skills and abilities of building interpersonal interaction.

\section{Conclusions}

In the sample of employees of a multi-ethnic environment, the positive ethnic identity of an individual was the most informative. The great number of statistically significant connections of personality traits and types of behavior was found out. Such characteristics of employees' personality as empathy and ethnic isolation (two correlations) were also quite informative. On the whole, there are more statistically significant correlations between personal and behavioral characteristics in the sample of employees in a multi-ethnic environment than in a mono-ethnic environment. The development of interpersonal interaction in a multi-ethnic environment in teams of employees in the period of reorganization should be based on the development of the most significant characteristics of their personality and behavior: the positive ethnic identity, empathy, interpersonal trust and skills of building interpersonal interaction.

The greatest problems in intercultural communication among team members and conflicts are caused by cultural differences. The solution to these problems depends on the use of cross-cultural 
management strategies. In order to form an effective management system of a multinational company, the following points should be taken into account: the type of multinational company, the influence of corporate and national cultures, the dominant position of one national culture in the team over others, the necessity to acculturate new members of the organization, training managers of multinational companies to work within a different national culture, being aware of a communication code of this foreign culture (the language, customs and rules of behavior, psychology and mentality, etc.).

\section{Acknowledgements}

Author Contributions: (V.K.) Vsevolod Konstantinov and (E.Sh.) Evgeniia Shumilkina conducted the empirical part of the research as well as analyzed and described the received data. (R.O.) Roman Osin provided significant assistance in interpreting the results and presenting them in the written form. All the above mentioned authors have made a considerable contribution to this work.

The study was conducted according to the American Psychological Association's (APA) ethical principles of psychologists and code of conduct.

Conflicts of Interest: The authors declare no conflict of interest.

\section{References}

Allen, T. D., \& Eby, L. T. d. T. (2012). The study of interpersonal relationships: An introduction. In L. T. d. T. Eby \& T. D. Allen (Eds.), SIOP organizational frontiers series. Personal relationships: The effect on employee attitudes, behavior, and well-being (pp. 3-13). Routledge/Taylor \& Francis Group.

Berry, J., Galyapina, V., Lebedeva, N., Lepshokova, Z., \& Ryabichenko, T. (2019). Intercultural Relations in Georgia and Tajikistan: A Post-Conflict Model. Psychology. Journal of the Higher School of Economics, 16(2), 232-249. doi:10.17323/1813-8918-2019-2-232-249

Bono, J. E., \& Yoon, D. J. (2012). Positive supervisory relationships. In L. T. d. T. Eby \& T. D. Allen (Eds.), SIOP organizational frontiers series. Personal relationships: The effect on employee attitudes, behavior, and well-being (pp. 43-66). Routledge/Taylor \& Francis Group

Dutton, J.E. (2014). Build high quality connections. In J.E. Dutton \& G.M. Spreitzer (Eds.), How to be a positive leader: Small actions big impact (pp. 11-21). San Francisco, CA: Berrett-Khoeler.

Grigoryev, D., van de Vijver, F., \& Batkhina, A. (2018). Discordance of Acculturation Attitudes of the Host Population and their Dealing with Immigrants. Journal of Intercultural Communication Research, 47(6), 491-509. doi:10.1080/17475759.2018.1497678

Hall, E. T. \& Hall, M. R. (1990). Understanding cultural differences: Germans, French and Americans. Yarmouth, ME: Intercultural Press.

Hofstede, G. H., Hofstede, G. J., \& Minkov, M. (2010). Cultures and organizations: Software of the mind. Maidenhead: McGraw-Hill.

Khukhlaev, O. E., Alexandrova, E. A., Gritstnko, V. V., Konstantinov, V. V., Kuznetsov, I. M., Pavlova, O. S., ... Shorokhova, V. A. (2019). Religious Group Identification and Ethno-National Attitudes in Buddhist, Muslim and Orthodox Youth. Cultural-Historical Psychology, 15(3), 71-82. doi:10.17759/chp.2019150308

Khukhlaev, O.E., Kuznetsov, I.M., \& Chibisova M.Yu. (2013). The integration of migrants in the educational environment: social and psychological aspects. Psychological Science and Education, 18 (3), 5-17.

Kluckhohn, F. R. \& Strodtbeck, F. L. (1961). Variations in value orientations. Evanston, IL: Row, Peterson.

Konstantinov, V. \& Kovaleva, N.A. (2013). Parting with motherland as socio-psychological problem of migration. Psychological Journal, 34(5), 3-15.

Konstantinov, V. (2017). The Role of the Host Local Population in the Process of Migrants' Adaptation. Social Sciences, 6(3), 92. doi: 10.3390/socsci6030092

Lewis, R. D. (2000). When cultures collide: managing successfully across cultures (2rd ed.). London: Nicholas Brealey Publishing.

Raman, P., Sambasivan, M., \& Kumar, N. (2016). Counterproductive work behavior among frontline government employees: Role of personality, emotional intelligence, affectivity, emotional labor, and emotional exhaustion. Journal of Work and Organizational Psychology, 32(1), 25-37. doi:10.1016/j.rpto.2015.11.002

Raygorodsky, D.Ya. (2017). Practical psychodiagnostics. Methods and tests. Samara, Bakhrakh-M Publ.

Ryzhova S., Konstantinov V., Gritsenko V. \& Khukhlaev O. (2018). Structure of religious identity of modern orthodox youth. Psychological Journal, 39(4), 95-104. doi:10.31857/S020595920000074-7

Tatarko, A., \& Lebedeva, N. (2011). Methods of ethnic and cross-cultural psychology. Moscow: HSE Publishing House. doi:10.17323/978-5-7598-0867-1 